\title{
Laparoscopic Choledochal Cyst Resection with Simplified Common Bile Duct Reconstruction in an Adult Population:
} \section{A Case Series}

\author{
Daniel Gomez ${ }^{1}$, Luis F Cabrera ${ }^{2}$, Mauricio Pedraza ${ }^{3}$, Sebastian Sanchez ${ }^{4}$, Jean A Pulido ${ }^{5}$, Ricardo Villarreal ${ }^{6}$, \\ Andres C Mendoza ${ }^{7}$, Daniela Moreno ${ }^{8}$, Natan Zundel ${ }^{9}$
}

\begin{abstract}
Introduction: Choledochal cysts (CC) are rare congenital pathology in adult population. Since 1995, laparoscopic management has been described for this entity. Nevertheless, its management is considered to be a controversial matter due to the augmented risk of associated cholangiocarcinoma.

Materials and methods: A retrospective, observational, and descriptive study was conducted considering patients diagnosed with CC who were operated at a hepatobiliary surgery referral center from January 2013 to June 2018. Patients were taken to simplified laparoscopic hepaticojejunostomy with a Roux-en-Y reconstruction. A retrospective analysis of the data obtained is presented.

Results: Ten adult patients with CC underwent surgical biliary reconstruction at a mean age of 34.5 years; $75 \%$ had Todani type I CC and $25 \%$ Todani type IV-B CC. About $50 \%$ of the patients were diagnosed via endoscopic retrograde cholangiopancreatography (ERCP) and $50 \%$ of them via magnetic resonance cholangiopancreatography. None required re-intervention, no mortality was reported; and the mean hospital stay was 5 days, no patient had postoperative biliary leakage, none was converted to open surgery, and all patients had adequate oral feeding tolerance 2 days postoperative. Long-term follow-up showed no incidence of cholangiocarcinoma after 2-year follow-up.

Conclusion: Choledochal cysts in adults is a rare pathology that has a high probability of developing malignancy when not adequately surgically managed and because of secondary bile reflux. These factors make surgical management a critical decision. The simplified laparoscopic approach presented in this paper seems to be an effective and safe alternative to biliary duct reconstructive surgery.

Keywords: Biliary duct surgery, Biliary reconstruction, Choledochal cyst, Laparoscopic approach.

World Journal of Laparoscopic Surgery (2019): 10.5005/jp-journals-10033-1366
\end{abstract}

\section{INTRODUCTION}

Choledochal cysts are congenital dilatations of the intrahepatic or extrahepatic biliary ducts. ${ }^{1-5}$ They are associated with the development of cholangiocarcinoma. Their incidence predominates in eastern population where it is reported to be approximately $0.00769 \%$ higher than western population where the incidence is around $0.000666 \%$. These are estimated numbers; the real incidence of CCs in adult population has not been established so far, since in most cases they are incidentally diagnosed on images or during surgery. ${ }^{1,2,6}$

The first case of laparoscopic approach for CC resection was published by Farello et al. in 1995, since then, different studies have shown that minimally invasive techniques can reduce surgical time, postoperative morbidity, postoperative pain control, and incidence of surgical site infections (SSI). Better results with laparoscopy are reported on patients who have CC Todani type I, II, and IVb compared with the results with open approaches. ${ }^{2,6,7}$

We present our experience with laparoscopic CC resection and simplified bile duct reconstruction in an adult population, analyzing the technical aspects of a novel procedure, postoperative clinical evolution of the patients involved in the study, and the incidence of cholangiocarcinoma in a 2-year postoperative follow-up.

\section{Materials and Methods \\ Design}

We conducted a retrospective, observational, and descriptive study with a population of 10 adult patients diagnosed with CC who
${ }^{1}$ Department of Advanced Laparoscopic Surgery, Universidad Militar Central, Bogotá, Colombia

${ }^{2}$ Department of General Surgery, Universidad El Bosque, Bogotá, Colombia; Department of General Surgery, Fundacion Santa Fe de Bogotá, Bogotá, Colombia

3,5,7,8 Department of General Surgery, Universidad El Bosque, Bogotá, Colombia

${ }^{4}$ Department of General Surgery, Pontificia Universidad Javeriana, Bogotá, Colombia

${ }^{6}$ Department of General Surgery, Universidad El Bosque, Bogotá, Colombia; Department of Gastrointestinal Surgery, Los Cobos Medical Center, Bogotá, Colombia

${ }^{9} \mathrm{FIU}$ Herbert Wertheim College of Medicine, Miami, Florida, USA; Minimally Invasive and Bariatric Surgery, Fundacion Santa Fe de Bogota University Hospital, Bogotá, Colombia

Corresponding Author: Mauricio Pedraza, Department of General Surgery, Universidad El Bosque, Bogotá, Colombia, Phone: +57 301370957, e-mail: mpedraza93@gmail.com

How to cite this article: Gomez D, Cabrera LF, Pedraza M, et al. Laparoscopic Choledochal Cyst Resection with Simplified Common Bile Duct Reconstruction in an Adult Population: A Case Series. World J Lap Surg 2019;12(2):76-82.

Source of support: Nil

Conflict of interest: None

(-) The Author(s). 2019 Open Access This article is distributed under the terms of the Creative Commons Attribution 4.0International License (https://creativecommons. org/licenses/by-nc/4.0/), which permits unrestricted use, distribution, and non-commercial reproduction in any medium, provided you give appropriate credit to the original author(s) and the source, provide a link to the Creative Commons license, and indicate if changes were made. The Creative Commons Public Domain Dedication waiver (http://creativecommons.org/publicdomain/zero/1.0/) applies to the data made available in this article, unless otherwise stated. 
underwent laparoscopic CC resection and simplified common bile duct reconstruction at a hepatobiliary referral center in Bogota, Colombia between January 2013 and June 2018.

The following variables were evaluated: age, sex, CC type (according to Todani classification), diagnosis, surgery time, bleeding, biliary leakage, conversion rates, oral feeding tolerance, Intensive care unit (ICU) stay, hospital stay time, need of reintervention, incidence of stenosis, mortality and incidence of cholangiocarcinoma with a postoperative follow-up of at least 2 years.

All patients had a pre-surgical multidisciplinary team assessment performed by gastroenterology and general surgery. Patient consent for laparoscopic choledochal cyst resection and simplified common bile duct reconstruction was obtained before the procedure was started. The study protocol was approved by the ethics committee at our institution and it was implemented in accordance with the Declaration of Helsinki and good clinical practice guidelines.

\section{INDICATIONS}

In order to include patients in this study, the following criteria were evaluated. Only patients over 18 years of age, who had a diagnosis of CC (Todani type I, II, and IVb) (Fig. 1) and Table 1 who were taken to laparoscopic CC resection and simplified common bile duct reconstruction were selected. Patients with the following characteristics were excluded from the study: those diagnosed with CC type III, IVa, V, and Child Pugh B (or higher) hepatic cirrhosis,
Table 1: Todani classification of the bile duct cyst

\begin{tabular}{ll}
\hline Type & Characteristics \\
\hline I & Solitary extrahepatic cyst \\
II & Extrahepatic diverticulum \\
III & Intraduodenal diverticulum (Choledochocele) \\
IVA & Extra- and intrahepatic cyst \\
IVB & Multiple extrahepatic cyst \\
V & Multiple intrahepatic cyst (Caroli's disease) \\
\hline
\end{tabular}

extrinsic bile duct compression (e.g., periampullary tumors), or cholangiocarcinoma, patients who had already been treated surgically for CC at another center, patients who were coursing with cholangitis and patients had a contraindication for laparoscopic surgery.

\section{Surgical Technique Patient Preparation}

All patients prepared for laparoscopic choledochal cyst resection and simplified common bile duct reconstruction were preparer as if they had an open operation, 8-hour fasting and antibiotic prophylaxis were indicated according to the latest good clinical practice guidelines. Patients and their families were informed of the surgical risk, possible complications, such as bleeding, infection, the need for additional trocars, open conversion, and mortality.
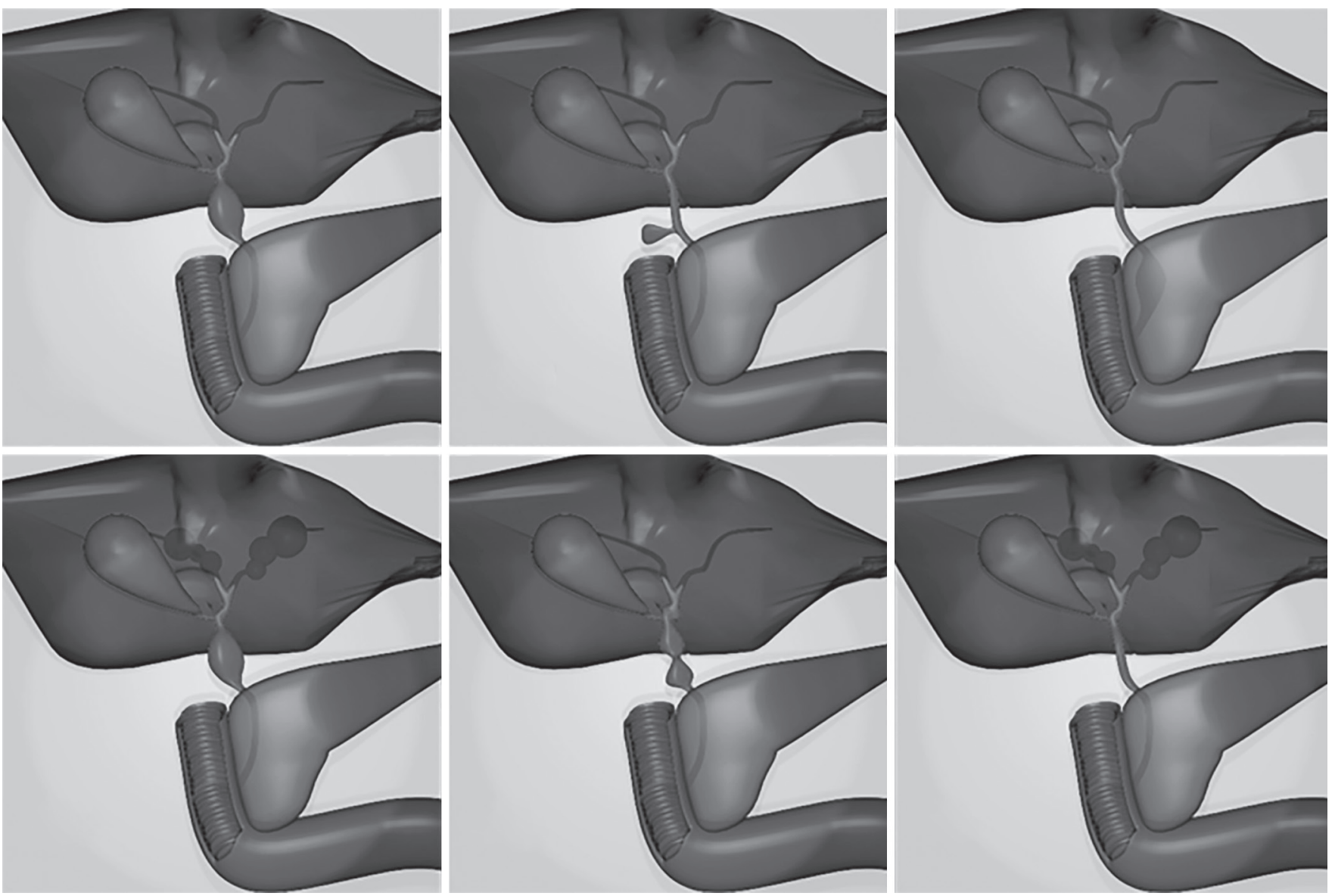

Fig. 1: Todani classification in 3D-model by Pulido $J$ 


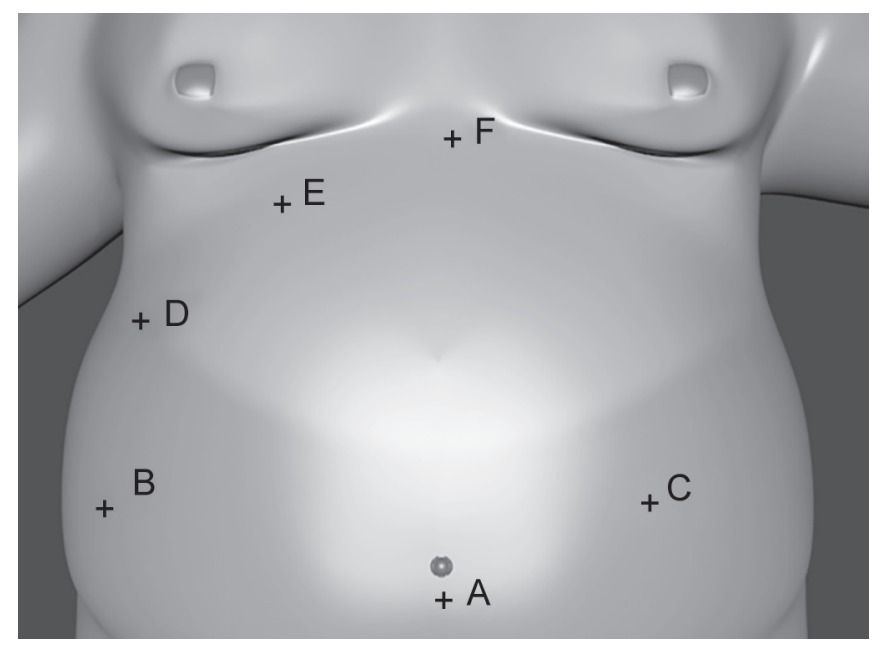

Figs $2 \mathrm{~A}$ to F: Surgical port sites (A) To applied pneumoperitoneum using an open Hasson umbilical approach; (B and C) $12 \mathrm{~mm}$ port; (D, E, and F) Are optional ports and could be used at $5 \mathrm{~mm}$ port

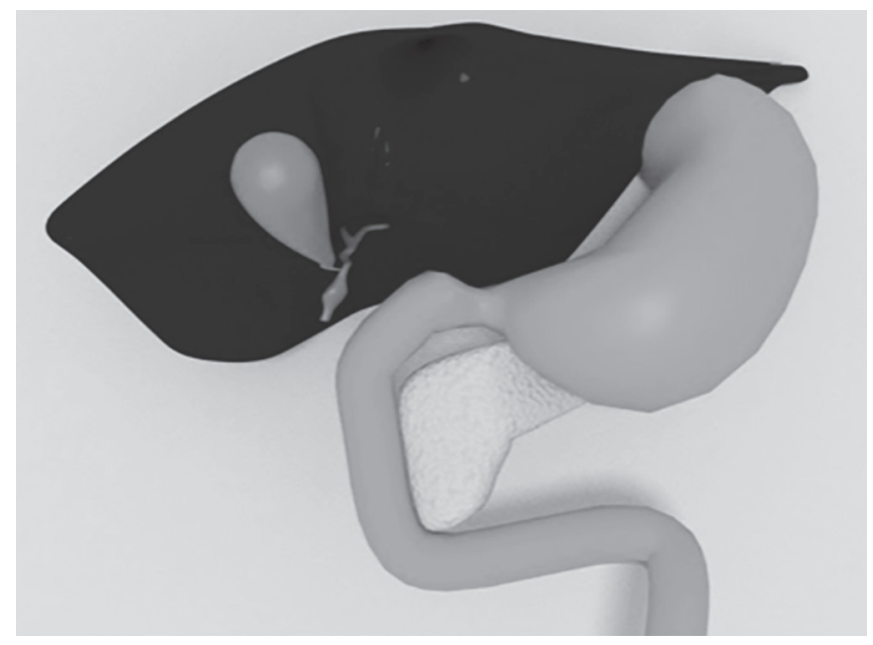

Fig. 4: Section of the choledochal cyst-distal portion

\section{Equipment and Room Set-up}

Under general anesthesia, all patients were placed in the supine position with both arms tucked along their sides with their legs spread wide open. The patients were securely strapped to the surgical bed to facilitate maximum tilting and lateral rotation of the surgical table. The surgeon positioned himself between the patient's legs in the French laparoscopy position. The first surgical assistant stood at the surgeon's right-hand side and the second assistant to the left. The scrub nurse stood to the right of the first surgical assistant.

\section{Laparoscopic Choledochal Cyst Resection and Simplified Common Bile Duct Reconstruction}

Using an open umbilical approach, a $12 \mathrm{~mm}$ port was introduced into the abdominal cavity in order to create a pneumoperitoneum with carbon dioxide maintaining an intra-abdominal pressure of $14 \mathrm{~mm} \mathrm{Hg}$ (Fig. 2). Under direct laparoscopic vision, using a $30^{\circ}$ lens, 4 additional ports were placed; two $12 \mathrm{~mm}$ ports, one in the right flank and the other in the left paramedial zone. The other two $5 \mathrm{~mm}$ ports were placed, one in the right upper quadrant, and the other one in the epigastrium. The port site placement for laparoscopic

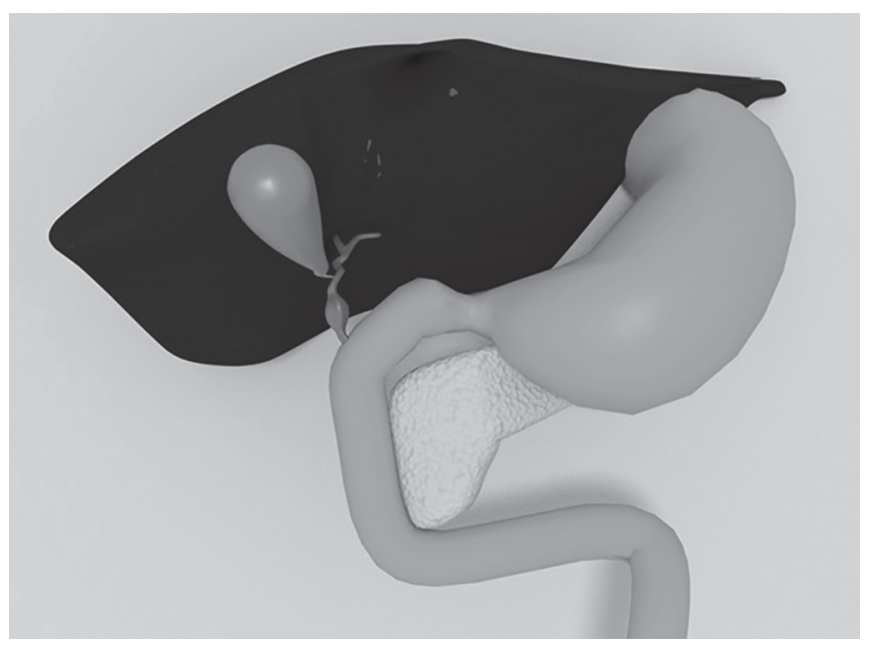

Fig. 3: Bilioenteric anatomy with CC type I

CC resection with simplified common bile duct reconstruction is demonstrated in Figure 3.

The procedure started with full dissection of the structures within the Calot's triangle using an ultrasonic laparoscopic cutter (Harmonic, Ethicon Endo Surgery Inc., Cincinnati, $\mathrm{OH}$, USA) from left to right exposing, cystic duct, artery, and vein. Dissection of intra-abdominal fat continued to expose other structures in order to better identify the common bile duct with the CC, hepatic artery, and portal vein. A Penrose drain was placed around the cyst to assist with retraction. The CC distal portion was sectioned less than $1 \mathrm{~cm}$ from the duodenum where the duct size was normal, this was done with an endoscopic mechanical linear cutter suture (The Endo GIA ${ }^{\mathrm{TM}}$ reinforced reload with Tri-Staple ${ }^{\mathrm{TM}}$ technology) (Fig. 4). After this, a partial laparoscopic cholecystectomy was performed, cutting only the cystic artery and maintaining the integrity of the cystic duct with an abandoned gallbladder. The proximal section of the common bile duct was made with laparoscopic scissors $1 \mathrm{~cm}$ away from the CC superior edge. Then, the gallbladder was extracted along with the cystic duct and CC. The extracted pieces were sent to the anatomic and pathological examination.

Longitudinal division of the greater omentum was performed allowing intestinal ascension for anastomosis. The Treitz ligament was identified and at $60-70 \mathrm{~cm}$ from it an omega loop was made and ascended, in an antecolic position, to the hepatic duct. A lateral-terminal hepatic-jejunostomy anastomosis was made using a Hepp-Couinaud approach without tension using simple non-continuous sutures with polydioxanone 4-0 (PDS, Ethicon, Inc., Cincinnati, OH, USA) (Fig. 5).

Tissue approximation was performed using a posterior initial suture continuing anteriorly, apply only the necessary number of sutures to prevent leakage ischemia, and stenosis. Around $100-150 \mathrm{~cm}$ distal from the anastomosis, a second omega loop was made with the intestinal tube from the duodenum (bile loop) and lateral-lateral jejunum-jejunum anastomosis was made $5 \mathrm{~cm}$ from the previous anastomosis using an endoscopic mechanical linear $60 \mathrm{~mm}$ suture (Fig. 6).

The subsequent wall was sutured using invaginating noninterrupted stitches with polydioxanone 3-0 (PDS, Ethicon, Inc., Cincinnati, $\mathrm{OH}, \mathrm{USA})$. The mesenteric defect was closed using simple non-interrupted sutures with polyester 2-0 (Ethibond, Ethicon, Inc., Cincinnati, $\mathrm{OH}, \mathrm{USA}$ ). The portion of the small intestine left between the two anastomoses was then separated using an endoscopic mechanical linear $60 \mathrm{~mm}$ suture leaving a Roux-en-Y configuration (Fig. 7). A 19 French round Blake drain was placed under the liver. 


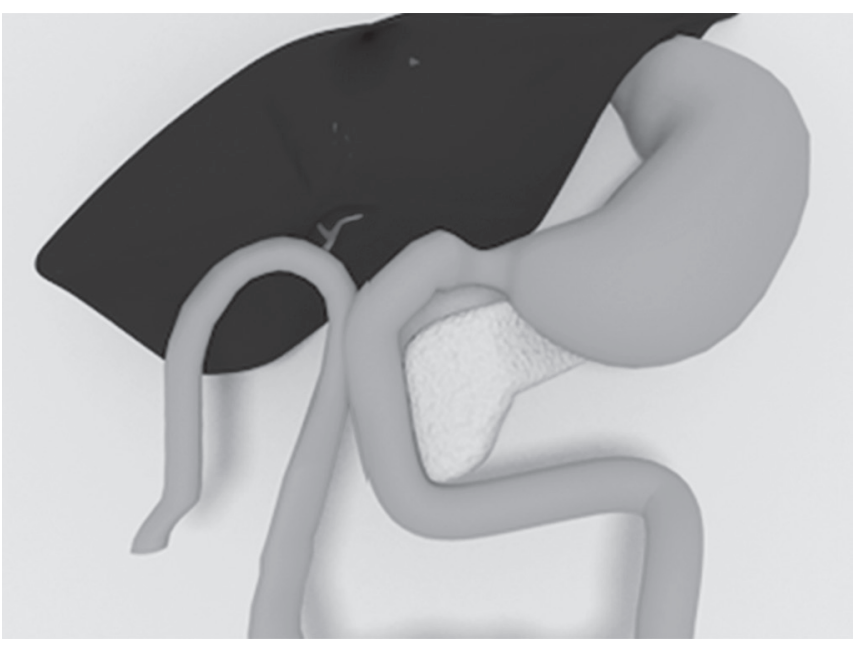

Fig. 5: Laparoscopic hepaticojejunostomy

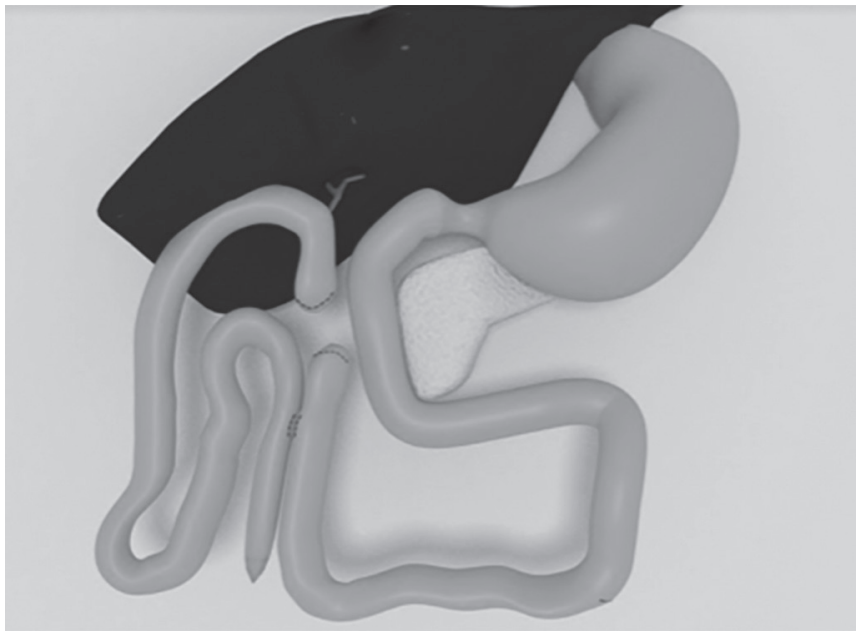

Fig. 7: Simplified hepaticojejunostomy in Roux-en-Y

Please refer to the online video material: Choledochal cyst 69556 Bestoun Ahmed (this material was presented at SAGES annual meeting, Baltimore, 2019).

\section{Postoperative Care}

The operative time was approximately 147 minutes. The patient ambulated on the same day of the procedure and tolerated oral food intake. The peritoneal drain was removed on postoperative day 2; and all the patients were discharged the following day. No patients required ICU or reintervention.

\section{Statistical Analysis}

Being an observational retrospective cohort, a descriptive analysis of the data was performed prospectively in Microsoft Excel databases and analyzed using SPSS1 (Statistical Package for The Social Sciences) V.22.0. Variables were analyzed and median, minimum, maximum values, and percentages were obtained.

\section{Results}

\section{Patient Characteristics}

Between 2013 and 2018, 10 patients with a diagnosis of CC underwent simplified laparoscopic bile duct reconstruction in

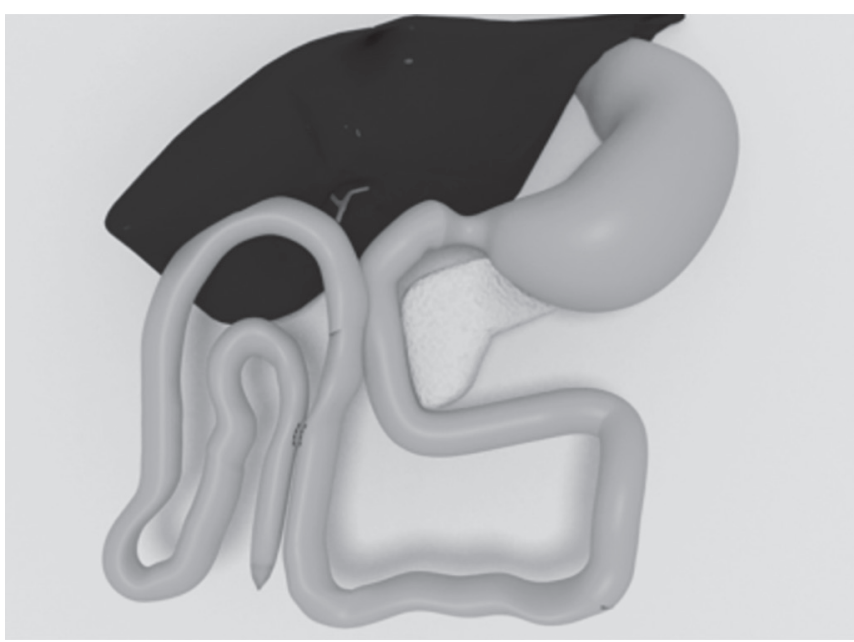

Fig. 6: Lateral-lateral omega jejunojejunostomy

Table 2: Patient referral characteristics with bile duct injury

\begin{tabular}{lll}
\hline Variable & Sub & $n=10(\%)$ \\
\hline Age (years, median, SD) & & 34.5 \\
Gender & Female & 100 \\
& Male & 0 \\
Choledochal cyst type & IA & 75 \\
& IVB & 25 \\
ASA classification & ASA 1/2 & 100 \\
Patients with preoperative ERCP or & ASA 3/4 & 0 \\
CPRMN & Yes & 100 \\
\hline ASA, & No & \\
\hline
\end{tabular}

ASA, the American Society of Anesthesiologists physical status classification

our center. Patients demographics are presented in Table 2. All patients were women, 75\% of them presented Todani type I CC and $25 \%$ Todani IV-B, without other associated malformations. About $50 \%$ of them were diagnosed during endoscopic retrograde cholangiopancreatography (ERCP) and other $50 \%$ with a magnetic resonance imaging (MRI). All of them were taken to resection of the common bile duct with a Roux-en-Y hepaticojejunostomy.

\section{Surgical Outcomes}

Average surgery time was 147 minutes; and the average intraoperative bleeding was $15 \mathrm{cc}$. No intra or postoperative complications were detected. There were no operative site infections, biliary leaks, and no mortality. The mean hospital stay time was 5 days.

One patient required ICU admission for 2 days due to anesthetic complications. There was no need for open conversion of the procedures and all patients had oral feeding and adequate tolerance at 1 day postoperative. Long-term follow-up showed no bile reflux at 2 years following surgery (Table 3 ).

\section{Discussion}

In 1723, Vater and Ezler described for the first time, the CC; however, it was not until 1977 that Todani described the classification that it is most frequently used nowadays (Fig. 1). 4,5,8,9 The management of this pathology has evolved from a cysto-enterostomy to advanced bilioenteric reconstructions with Roux-en-Y configuration. 3,10 
Laparoscopic Choledochal Cyst Resection with Biliary Reconstruction

Table 3: Surgical variables and outcomes, with at least 2-year follow-up

\begin{tabular}{ll}
\hline Variable & $(\%), S D$ \\
\hline Average surgery time & 147 minutes \\
& $(113-195$ minutes $)$ \\
Average intraoperative bleeding & $15-50 \mathrm{cc}$ \\
Mean hospitality stay & 5 days \\
Bile leaks & 0 \\
Converted to open surgery & 0 \\
Oral feeding and adequate tolerance at POP & 2 \\
(days) & \\
Mortality & 0 \\
\hline
\end{tabular}

POP, postoperative

Prior to 1980's, management for common bile duct consisted on drainage; however, reports of cholangiocarcinoma, recurrent cholangitis, and biliary lithiasis led to a change in the way surgeons managed this disease. ${ }^{3,4}$ That is why currently, the management of Todani I, II, and IV CC is similar and involve a complete resection of the defect with posterior bilioenteric reconstruction. ${ }^{1-3,5,11}$

Sastry et al. show the incidence of CC according to the Todani classification, being type I the most frequent (69.8\%), followed by type IV (23.7\%), type V (3.1\%), type II (2\%) and type III (1.4\%). ${ }^{12}$ The goal of the surgical management of type IV-A and IV-B CC is to stop or at least slow down the progression of liver damage. The complications of non-operated CC are the result of stasis in which cholangitis, biliary stone formation, recurrent pancreatitis, cirrhosis, and portal hypertension without mentioning the risk for cholangiocarcinoma; for that reason, surgical management in adult population is indicated. ${ }^{13}$

When complete resection of the cyst is not possible (usually in CC type IV-A) complete resection of the extrahepatic biliary tract should be performed in addition to a lobectomy (of the compromised portion of the intrahepatic biliary tree) with posterior biliary-enteric reconstruction. Incomplete resection of the cyst does not seem to be related to perioperative complications although some argue a persistent risk of malignancy. ${ }^{14,15}$

The usefulness of laparoscopy in the surgical management of this type of patients has been questioned due to the complexity of the procedures, the need for precise movements, and the long learning curve necessary to obtain adequate results. ${ }^{16}$ However, resection with minimally invasive technique with a Roux-en- $Y$ reconstruction has been shown to be safer when compared to open approach. ${ }^{16,17}$

Some of the advantages of laparoscopic management in these cases are the better visualization of structures, more precise dissections due to the magnification of structures, less postoperative pain, shorter hospital stay time, better esthetic results, decreased bleeding, lower risk SSI and lower incidence of postoperative ileus; being the only negative aspect a prolonged surgical time. ${ }^{6,18}$ However, some authors have described an association between the laparoscopic approach and an augmented risk of malignancy due to incomplete $\mathrm{CC}$ resections that lead to chronic inflammatory process; they argue that open approach should not be fully abandoned since it is possible to better identify structures even in cases of severe local inflammatory processes and anatomical distortion. ${ }^{3,11}$

Some patients benefit from a two-stage surgical procedure, where during the first surgical time the CC is drained. During the second operative time (after physiological resuscitation in the
ICU and resolution of the acute phase), the resection of the cyst is performed. In our series of cases, complete resection of the CC and bilioenteric reconstruction was performed in one surgical time with a $0 \%$ conversion rate and no need of reintervention.

Perhaps the most important aspect of the procedure, besides an adequate resection of the $\mathrm{CC}$, is an adequate biliary-enteric reconstruction where the anastomosis made should allow free biliary flow into the intestine, avoiding as biliary reflux, which is the most important cause malignancy in these patients. ${ }^{1,3}$ It can be done with a hepatic-duodenostomy, choledochojejunostomy, or with a Roux-en-Y hepaticojejunostomy. ${ }^{1,3,6}$ Being the first and third the most frequent; today there is great controversy regarding which of the two most used procedures is the best as it can be evidenced in the experience of Narayanan et al., who in 2013 published a systematic review in which when comparing 679 cases of patients, $60.7 \%$ taken to hepatico-duodenostomy and $39.3 \%$ to hepaticojejunostomy, respectively, they reported: a hospital stay time of 4.8 days and 6.1 days, the incidence of biliary leakage $2.1 \%$ and $2.94 \%$, the incidence of cholangitis $2.47 \%$ and $2.42 \%$, the incidence of anastomotic stenosis $1.21 \%$ and $1.47 \%$, the incidence of biliary reflux $5.88 \%$ and $0 \%$, incidence of intestinal obstruction due to adhesion syndrome $0 \%$ and $5.12 \%$, and the need for re-intervention $1.21 \%$ and $2.45 \% .{ }^{19}$ Most studies demonstrate that there are really no clinically significant differences between the two procedures. However, due to the existing evidence with hepaticoduodenostomy and the development of gastric cancer secondary to biliary reflux, in our study, we only performed bilioenteric reconstruction with a Roux-en-Y hepaticojejunostomy. ${ }^{1,20}$ In our case series, there was no biliary leakage, no re-intervention were needed; and our hospital stay time was shorter to the one reported in the medical literature.

It is a common factor among the opinion of experts in the world medical literature on this regard that the laparoscopic approach is a challenging for the surgeon but if performed correctly it is effective and appropriate. ${ }^{2}$ The conversion rate to open approach ranges from $0 \%$ to $37 \%$, as described by Palanivelu et al. (2008) in a study published in the Journal of the American College of Surgeons that report a conversion rate of $8.5 \%$. However, in our series there were no conversions to open surgery. ${ }^{3}$ The age of the patient has been shown to be directly related to the need for conversion, is it more frequent in pediatric patients than in adults, which is extrapolated to our results. ${ }^{2}$ On average, the length of hospital stay time in minimally invasive management ranges from 3 days to 4.7 days and in the open approach from 5 days to 20.5 days and has a mortality of up to $3.3 \%$, as evidenced in our series. ${ }^{2,3,6}$

Early postoperative complications include pancreatitis, enteric or biliary leakage from the anastomosis, bleeding, SSI, and pancreatic or biliary fistulas. The most frequent delayed complications (after 30 days postoperative) are intrahepatic or extrahepatic bile duct stenosis, lithiasis, malignancy, intestinal obstruction, recurrent pancreatitis, hepatic cirrhosis, and cholangitis. ${ }^{1,4,8}$ Postoperative complications in children are rare; however, in adults, they occur between $17 \%$ and $40 \%$ of cases. ${ }^{1}$ the most frequent complication with an incidence of $0 \%$ to $20 \%$ of cases is an anastomotic leakage; however, in our study, there were no postoperative complications. ${ }^{3}$

Studies that support the use of robots for the surgical management of CC, such as the one reported by Wang et al. who reported their experience in 2016 with a 26 -year-old patient who was diagnosed with a type I CC and decided to take him to a robotic Roux-en-Y hepaticojejunostomy with the alimentary and bilioenteric loops in a retrocolic position with satisfactory results in terms of incidence, early or late complications, esthetic results 
and SSI, with a surgical time of 480 minutes (longer when compared with our surgical time) and a bleeding of $100 \mathrm{cc}$ (also greater than the one reported in our series). ${ }^{10}$ The evidence on robotic surgery in these cases is very limited; however, some authors consider that aspects such as the magnified three-dimensional image, along with more precise movements could represent the difference in the prognosis of the patients. ${ }^{1,10}$

As it was said before, some authors have reported that cholangiocarcinoma may be a secondary to unresected portions CC, with an incidence of up to $30 \%$ in the adult population. ${ }^{26}$ The global incidence reported in the word medical literature for cholangiocarcinoma is 0.95 per 100,000 , being more frequent in patients with CC. ${ }^{21,22}$ The risk of malignancy reported for these patients is $0.7 \%$ to $28 \%$, this risk increases over time. ${ }^{2,5,6,8,23}$ Sastry et al., in one of their studies with a population of 7,880 patients (1,914 under 18 years of age and 3,866 adults), from which $4.59 \%$ had histopathological studies revealing malignancy, $70.4 \%$ of these patients had cholangiocarcinoma, 23.5\% gallbladder carcinoma, and $6.1 \%$ other malignancies. The incidence of cholangiocarcinoma in type I CC is $68 \%$; and in type IV CC it is of $21 \% .{ }^{21}$ The risk of malignancy is high, and it is associated with a global survival of 6 to 21 years. Incidence of malignancy referred by Amid et al., in type IV CC was of $9.2 \%$, type I $7.6 \%$, type II $4.3 \%$, type III $4 \%$, and type $\mathrm{V} 2.5 \%$. ${ }^{12}$ For this reason, it is imperative to make a complete resection of CC and to make a strict postoperative follow-up.

There is still some controversy regarding abandoned CC parts and their risk of malignancy; however, some of the studies on this regard seem to indicate that a complete resection is superior to a partial resection in terms of reducing the risk of malignancy as it is shown by Ten Hove et al. in a meta-analysis published in 2018 , where they compare a total of 80 studies and 2,904 patients, with an incidence of $10.7 \%$ of cholangiocarcinoma, with an increased risk for patients with type I and IV CC with a statistically significant $p$ of 0.016 , and with an increased risk for cholangiocarcinoma for patients taken to CC drainage and partial resection with an OR of 3.97, almost four times more than patients taken to complete resection (as it was performed in our series). ${ }^{1,2,24,25}$ Mutations in K-ras and $p 53$ genes have been associated with cholangiocarcinoma and a history of common bile duct disease. ${ }^{1,4,8}$ Nevertheless, no tumor markers show direct relationship between their serum levels and the presence of cholangiocarcinoma. ${ }^{26}$

Nicholl et al. revealed a direct correlation between patient age and cancer risk: from 0 year to 30 years (0\%), from 31 years to 50 years (19\%), and from 51 years to 70 years (50\%). ${ }^{27}$ Surgical management of CC with complete surgical excision during early life tends to decrease the probability of developing cancer cholangiocarcinoma later in life especially in the group of patients with type I CC. ${ }^{28,29}$ One study of 56 patients with a previous history of resection three patients progressed to cholangiocarcinoma with a range of 2-19 years after the procedure, showing that a strict follow-up is necessary after the procedure and during a long period of time to ensure an early diagnosis and improve long-term outcomes even in malignant positive patients. ${ }^{30}$ Other study that supports this shows that 15 patients out of 214 cases reported were associated with malignancy of the biliary tree; their survival was of 6-21 months after being diagnosed, poor prognosis was associated with late ${ }^{31}$ Lee et al. in a study of 40 patients diagnosed with cholangiocarcinoma during early stages and had a better prognosis; specifically, the 5 -year survival rates of patients with stages $\mathrm{la}, \mathrm{lb}$, and Ila cholangiocarcinoma reached $90.4 \%, 40.0 \%$, and $25.1 \%$, respectively. ${ }^{32}$ The main limitation of this study is that it is an observational retrospective study without randomization and no control group. It is therefore subject to selection bias. In addition, the population size of our study may be too small to draw statistically relevant conclusion, even if it is an infrequent disease in Latin America.

\section{Conclusion}

Adult CC are a group of rare entities that lead to high mortality and risk of developing cancer, these make their management critical and necessary. Complete resection of the CC and laparoscopic Rouxen-Y bilioenteric reconstruction is an effective and safe method, although complex and challenging for the surgeon. This simplified laparoscopic approach to bile duct reconstruction with Roux-en-Y anastomosis seems to be an effective and safe alternative for the management of this pathology. Additional research is warranted to assess long-term results in a larger series.

\section{Acknowledgments}

The authors are pleased to acknowledge Dr Ximena Alvira who provided clinical feedback and assistance in preparing the manuscript for submission. Dr Ernest Njeru provided quality English translation.

\section{References}

1. Soares KC, Arnaoutakis DJ, Kamel I, et al. Choledochal cysts: presentation, clinical differentiation, and management. J Am Coll Surg 2014;219(6):1167-1180. DOI: 10.1016/j.jamcollsurg.2014.04.023.

2. Hasan A, Nuha Y, Basil A. Laparoscopic resection of type I choledochal cyst in an adult and Roux-en-Y hepaticojejunostomy: a case report and literature review. Surg Laparosc Endosc Percutan Tech 2006;16(6):439-444. DOI: 10.1097/01.sle.0000213768.70923.99.

3. Palanivelu C, Rangarajan M, Parthasarathi $R$, et al. Laparoscopic management of choledochal cysts: technique and outcomes - a retrospective study of 35 patients from a tertiary center. J Am Coll Surg 2008;207(6):839-846. DOI: 10.1016/j.jamcollsurg.2008.08.004.

4. Edil BH, Olino K, Cameron JL. The current management of choledochal cysts. Adv Surg 2009;43:221-232. DOI: 10.1016/j.yasu.2009.02.007.

5. Tadokoro H, Takase M. Recent advances in choledochal cysts. OJ Gas 2012;2(4):145-154. DOI: 10.4236/ojgas.2012.24029.

6. Ahmed B, Sharma P, Leaphart CL. Laparoscopic resection of choledochal cyst with Roux-en-Y hepaticojejunostomy: a case report and review of the literature. Surg Endosc 2017;31(8):3370-3375. DOI: 10.1007/s00464-016-5346-3.

7. Crespo G, Garcia M, Marqués E, et al. Lesiones quísticas del conducto cístico (lesiones tipo VI). Rev Esp Enferm Dig 2017;109(5):373.

8. Soares KC, Goldstein SD, Ghaseb MA, et al. Pediatric choledochal cysts: diagnosis and current management. Pediatr Surg Int 2017;33(6): 637-650. DOI: 10.1007/s00383-017-4083-6.

9. Manoj Kumar GP, Rajagopalan B. Choledochal cyst. Med J Armed Forces India 2012;68(3):296-298. DOI: 10.1016/j.mjafi.2012.04.011.

10. Wang S-E, Chen S-C, Shyr B-U, et al. Robotic assisted excision of type I choledochal cyst with Roux-en-Y hepaticojejunostomy reconstruction. Hepatobiliary Surg Nutr 2017;6(6):397-400. DOI: 10.21037/hbsn.2017.01.15.

11. Stringer M. Laparoscopic management of choledochal cysts: is a keyhole view missing the big picture? Pediatr Serg Int 2017;33(6): 651-655. DOI: 10.1007/s00383-017-4089-0.

12. Sastry AV, Abbadessa B, Wayne MG, et al. What is the incidence of biliary carcinoma in choledochal cysts, when do they develop, and how should it affect management? World J Surg 2015;39(2):487-492. DOI: 10.1007/s00268-014-2831-5.

13. Chaturvedi A, Singh J, Rastogi V. Case report: cholangiocarcinoma in a choledochal cyst. Indian J Radiology \& Imaging 2008;18(3):236-238. DOI: $10.4103 / 0971-3026.41836$. 
14. Congo K, Lopes MF, Oliveira PH, et al. Outcomes of choledochal cysts with or without intrahepatic involvement in children after extrahepatic cyst excision and Roux en Y hepaticojejunostomy. Ann Hepatol 2012;11(4):536-543. DOI: 10.1016/S1665-2681(19)31468-1.

15. Shimamura K, Kurosaki I, Sato D, etal. Intrahepatic cholangiocarcinoma arising 34 years after excision of a type IV - a congenital choledochal cyst: report of a case. Surg Today 2009;39(3):247-251.

16. Tang Y, Li F, He G. Comparison of single-incision and conventional laparoscopic cyst excision and Roux en $\mathrm{Y}$ hepaticojejunostomy for children with choledochal cyst. Indian J Surg 2016;78(4):259-264. DOI: 10.1007/s12262-015-1348-y.

17. Urushihara N, Fukumoto K, Fukuzawa H, et al. Hepaticojejunostomy and intrahepatic cystojejunostomy for type IV - A choledochal cyst. J Pediatr Surg 2007;42(10):1753-1756. DOI: 10.1016/j.jpedsurg. 2007.06.012.

18. Lee $\mathrm{H}$, Hirose $\mathrm{S}$, Bratton $\mathrm{B}$, et al. Initial experience with complex laparoscopic biliary surgery in children: biliary atresia and choledochal cyst. J Pediatr Surg 2004;39(6):804-807. DOI: 10.1016/ j.jpedsurg.2004.02.018; discussion 804-7.

19. Narayanan SK, Chen Y, Narasimhan KL, et al. Hepaticoduodenostomy versus hepaticojejunostomy after resection of choledochal cyst: a systematic review and meta-analysis. J Pediatr Surg 2013;48(11): 2336-2342. DOI: 10.1016/j.jpedsurg.2013.07.020.

20. Madadi-Sanjani O, Petersen C, Ure B. Minimally invasive hepatobiliary surgery. Clin Perinatol 2017;44(4):805-818. DOI: 10.1016/j.clp. 2017.08.004.

21. Madadi-Sanjani O, Wirth TC, Kuebler JF, et al. Choledochal cyst and malignancy: a plea for lifelong follow-up. Eur J Pediatr Surg 2017;29(2):143-149. DOI: 10.1055/s-0037-1615275.

22. Drabek J, Keil R, Stovicek J, et al. The role of endoscopic retrograde cholangiopancreatography in choledochal cysts and/or abnormal pancreatobiliary junction in children. Prz Gastroenterol 2017;12(4):303-309. DOI: 10.5114/pg.2017.72107.
23. Ten Hove A, de Meijer VE, Hulscher JBF, et al. Meta-analysis of risk of developing malignancy in congenital choledochal malformation. $\mathrm{Br}$ J Surg 2018;105(5):482-490. DOI: 10.1002/bjs.10798.

24. Lal R, Agarwal S, Shivhare R, et al. Type IV-a choledochal cysts: a challenge. J Hepatobiliary Pancreat Surg 2005;12(2):129-134. DOI: 10.1007/s00534-004-0960-1.

25. Banks JS, Saigal G, D'Alonzo JM, et al. Choledochal malformations: surgical implications of radiologic findings. AJR Am J Roentgenol 2018;210(4):748-760. DOI: 10.2214/AJR.17.18402.

26. Katabi N, Pillarisetty VG, DeMatteo R, et al. Choledochal cysts: a clinicopathologic study of 36 cases with emphasis on the morphologic and the immunohistochemical features of premalignant and malignant alterations. Hum Pathol 2014;45(10):2107-2114. DOI: 10.1016/j.humpath.2014.06.016.

27. Nicholl $M$, Pitt HA, Wolf $P$, et al. Choledochal cysts in western adults: complexities compared to children. J Gastrointest Surg 2004;8(3): 245-252. DOI: 10.1016/j.gassur.2003.12.013.

28. Ong J, Campbell W, Taylor MA. Metastatic cholangiocarcinoma following choledochal cyst excision: an unusual cause of abdominal pain in a 35-year-old female. Ulster Medical Journal 2013;82(1):21-22.

29. Voyles CR, Smadja C, Shands WC, et al. Carcinoma in choledochal cysts. Age-related incidence. Arch Surg 1983;118(8):986-988. DOI: 10.1001/archsurg.1983.01390080088022.

30. Kobayashi S, Asano T, Yamasaki M, et al. Risk of bile duct carcinogenesis after excision of extrahepatic bile ducts in pancreaticobiliary maljunction. Surgery 1999;126(5):939-944. DOI: 10.1016/S00396060(99)70036-X.

31. He XD, Wang L, Liu W, et al. The risk of carcinogenesis in congenital choledochal cyst patients: An analysis of 214 cases. Annals of Hepatology 2014;13(6):819-826. DOI: 10.1016/S1665-2681(19)30985-8.

32. Lee SE, Jang JY, Lee YJ, et al. Choledochal cyst and associated malignant tumors in adults: a multicenter survey in South Korea. Arch Surg 2011;146(10):1178-1184. DOI: 10.1001/archsurg.2011.243. 